\title{
AN ABSTRACT LINEAR VOLTERRA EQUATION WTTH A NONCONVOLUTION KERNEL
}

\section{T. KIFFE}

\begin{abstract}
This paper is concerned with the existence and uniqueness of solutions to the equation $x(t)+\int_{0}^{t} a(t, \tau) A x(\tau) d r=f(t)$ where $A$ is an unbounded, positive, selfadjoint operator on a Hilbert space. A representation is given for the solution of this equation.
\end{abstract}

1. Introduction. In this paper we will be concerned with the existence and uniqueness of solutions to the equation

$$
x(t)+\int_{0}^{t} a(t, \tau) A x(\tau) d \tau=f(t), \quad 0<t<T,
$$

where $A$ is an unbounded, positive, selfadjoint operator on a Hilbert space $H$, $a(t, \tau)$ is a real-valued function and $x, f:[0, T] \rightarrow H$. Our goal is to extend the existence and uniqueness results of Clement and Nohel [1], Friedman and Shinbrot [2], and Kiffe and Stecher [7] for the convolution equation

$$
x(t)+\int_{0}^{t} b(t-\tau) A x(\tau) d \tau=f(t), \quad 0<t<T,
$$

to the nonconvolution equation (1.1).

Our approach to solving (1.1) will follow that of [1], [7] and consists of first considering the properties of the solutions of the resolvent scalar equations

$$
r_{\lambda}(t, \tau)+\lambda \int_{\tau}^{t} a(t, u) r_{\lambda}(u, \tau) d u=a(t, \tau)
$$

and

$$
s_{\lambda}(t, \tau)+\lambda \int_{\tau}^{t} a(t, u) s_{\lambda}(u, \tau) d u=1 .
$$

If we define resolvent operators $R(t, \tau)$ and $S(t, \tau)$ by

$$
R(t, \tau)=\int_{0}^{\infty} r_{\lambda}(t, \tau) d E(\lambda)
$$

and

$$
S(t, \tau)=\int_{0}^{\infty} s_{\lambda}(t, \tau) d E(\lambda)
$$

where $\{E(\lambda) \mid \lambda \geqslant 0\}$ is the resolution of the identity determined by $A$, it will be shown that solutions of (1.1) can be written in the form

$$
x(t)=f(t)-\int_{0}^{t} R(t, \tau) A f(\tau) d \tau,
$$

Received by the editors March 27, 1980.

AMS (MOS) subject classifications (1970). Primary 45D05, 45NO5; Secondary 47B25. 
or

$$
x(t)=S(t, 0) f(0)+\int_{0}^{t} S(t, \tau) f^{\prime}(\tau) d \tau
$$

under suitable assumptions on $a, f$, and $f^{\prime}$. For other related results on linear Volterra equations in abstract spaces we refer the interested reader to [5], [6].

2. Statement and discussion of results. Throughout this paper $\boldsymbol{H}$ will denote a real Hilbert space with norm $|\cdot|$ and inner product $\langle\cdot, \cdot\rangle$ and

$$
L^{1}[0, T ; H]=\left\{f:[0, T] \rightarrow H \mid f \text { is strongly measurable and } \int_{0}^{T}|f(t)| d t<\infty\right\} .
$$

$A$ will always denote an unbounded, positive, linear, selfadjoint operator from $H$ to $H$ with dense domain $D(A)$ and $\{E(\lambda) \mid \lambda>0\}$ will denote the resolution of the identity determined by $A$. For the standard results concerning the resolution of the identity and the spectral theorem for selfadjoint operators we refer the reader to [10]. We shall also set $H_{\alpha}=D\left(A^{\alpha}\right)$ for $0<\alpha<\infty$ and if we define a norm on $H$ by $\|x\|_{\alpha}=|x|+\left|A^{\alpha} x\right|$ then $H_{\alpha}$ becomes a Hilbert space itself.

Next we define precisely what we mean by a solution of (1.1). A function $x$ : $[0, T] \rightarrow H$ is a strong solution of (1.1) if $x \in L^{1}[0, T ; H], x(t) \in D(A)$ a.e. on $[0, T], A x \in L^{1}[0, T ; H]$ and $x(t)$ satisfies $(1.1)$ on $[0, T]$. Later we will define a weak solution of (1.1).

Concerning the kernel $a(t, \tau)$ we shall assume

(i) $a(t, \tau)$ is continuous for $0 \leqslant \tau \leqslant t \leqslant T$ and is absolutely continuous in $t$ for each fixed $\tau, \tau \leqslant t \leqslant T$;

(ii) $0<\varepsilon \leqslant a(t, \tau)$ for $0<\tau \leqslant t \leqslant T$ for some constant $\varepsilon$ and $(\partial / \partial t) a(t, \tau)<0$ for $0<\tau \leqslant t \leqslant T$;

(iii) $a(t, t)+\int_{0}^{t}(\partial / \partial t) a(t, \tau) d \tau>0$ for $0<t \leqslant T$;

(iv) for each $\lambda \geqslant 0$ the solution of (1.3) satisfies $r_{\lambda}(t, \tau)>0$ for $0<\tau<t<T$.

THEOREM 1. Suppose $a(t, \tau)$ satisfies (i)-(iv). If $f=f_{1}+f_{2}$ where $f_{1} \in$ $L^{1}\left[0, T ; H_{1+\alpha}\right]$ and $f_{2} \in W^{1,1}\left[0, T ; H_{\alpha}\right]^{\prime}$ for some $\alpha, 0<\alpha<1$, then (1.1) has a unique strong solution $x(t)=x_{1}(t)+x_{2}(t)$ where $x_{1}(t)$ is given by (1.7) with $f=f_{1}$ and $x_{2}(t)$ is given by (1.8) with $f=f_{2}$. Furthermore there is a constant $c=c(T)>0$ depending only on $a, \alpha$ and $T$ such that

$$
\|x\|_{L^{1}[0, T ; H]} \leqslant c\left\{\left\|f_{1}\right\|_{L^{1}\left[0, T ; H_{a}\right]}+\left\|f_{2}\right\|_{W^{1,1}[0, T ; H]}\right\} .
$$

In Theorem $1, W^{1,1}$ is the usual Sobolev space and concerning the hypothesis on $a(t, \tau)$ we remark that (i) and (ii) imply that for each $\lambda>0$ the solution of (1.4) satisfies $s_{\lambda}(t, \tau) \geqslant 0$ (see (3.2)-(3.4) below). A sufficient condition on $a(t, \tau)$ which insures (iv) is given in [4, Theorem 1] which under suitable differentiability conditions is equivalent to $(\partial / \partial \tau)(\partial / \partial t) \log a(t, \tau)<0$. Condition (iii) is a technical assumption needed to handle nonconvolution kernels. These assumptions on $a(t, \tau)$ are the natural extension to nonconvolution kernels of the conditions imposed on $b(t)$ in (1.2) in [1], [7]. In [2] Laplace transform methods are used to study (1.2) and hence their methods have no direct extension to convolution equations. Also in [1], [2] (1.2) is studied in a more general setting than used here for (1.1). 
Similar to [1] we define a weak solution of (1.1) as follows. A function $x$ : $[0, T] \rightarrow H$ is a weak solution of (1.1) if there are sequences $\left\{x_{n}\right\}$ and $\left\{f_{n}\right\}$ where each $f_{n} \in L^{1}[0, T ; H]$ and each $x_{n}$ is a strong solution of (1.1) with $f=f_{n}$ such that $f_{n} \rightarrow f$ and $x_{n} \rightarrow x$ in $L^{1}[0, T ; H]$. It is immediate from (2.1) that (1.1) has a unique weak solution if $f \in L^{1}\left[0, T ; H_{\alpha}\right]+W^{1,1}[0, T ; H]$ for some $\alpha, 0<\alpha<1$, given by (1.7) and (1.8). (Note that $L^{1}\left[0, T ; H_{1+\alpha}\right]$ is dense in $L^{1}\left[0, T ; H_{\alpha}\right]$ with respect to the norm in $L^{1}\left[0, T ; H_{\alpha}\right]$; similarly $W^{1,1}\left[0, T ; H_{\alpha}\right]$ is dense in $W^{1,1}[0, T ; H]$.)

If $f$ satisfies the hypotheses of Theorem 1 with $\alpha=1$ (i.e., $f \in$ $\left.L^{1}\left[0, T ; H_{2}\right]+W^{1,1}\left[0, T ; H_{1}\right]\right)$ then the hypotheses on $a(t, \tau)$ can be significantly weakened.

Theorem 2. Suppose $a(t, \tau)$ is positive and continuous for $0<\tau<t<T$, that for each fixed $\tau, a(t, \tau)$ is a nonincreasing function of $t$, and that $\int_{0}^{t} a(t, \tau) d \tau$ is absolutely continuous for $0 \leqslant t<T$. If $f \in L^{1}\left[0, T ; H_{2}\right]+W^{1,1}\left[0, T ; H_{1}\right]$ then (1.1) has a unique strong solution $x(t)=x_{1}(t)+x_{2}(t)$ where $x_{1}$ and $x_{2}$ are as given in Theorem 1 and $x(t)$ satisfies (2.1).

Theorem 2 now implies that (1.1) has a unique weak solution given by (1.7) and (1.8) if $f \in L^{1}\left[0, T ; H_{1}\right]+W^{1,1}[0, T ; H]$ if $a(t, \tau)$ is continuous, positive and nonincreasing in $t$. The proof of Theorem 2 uses a remarkable inequality due to Levin [8].

3. Proofs. Let $r_{\lambda}(t, \tau)$ and $s_{\lambda}(t, \tau)$ be the unique solutions of (1.3) and (1.4) respectively. By Theorem 3.1 of [9] $r_{\lambda}(t, \tau)$ and $s_{\lambda}(t, \tau)$ are continuous in $(t, \tau)$ for each fixed $\lambda$ and a direct application of Gronwall's inequality shows that $r_{\lambda}(t, \tau)$ and $s_{\lambda}(t, \tau)$ are also continuous in $\lambda$. Also a direct substitution establishes that

$$
s_{\lambda}(t, \tau)=1-\lambda \int_{\tau}^{t} r_{\lambda}(t, u) d u
$$

By hypothesis $r_{\lambda}(t, \tau)>0$ for $\lambda>0,0<\tau<t<T$. Next we show that

$$
s_{\lambda}(t, \tau)>0 \text { for } \lambda \geqslant 0,0<\tau<t<T \text {. }
$$

Fix $\tau$ and $\lambda$. Suppose (3.2) is false. Since $s_{\lambda}(\tau, \tau)=1$ there is a number $t_{0}>\tau$ so that $s_{\lambda}\left(t_{0}, \tau\right)=0$ but $s_{\lambda}(t, \tau)>0$ for $\tau<t<t_{0}$. Hence we must have that $(\partial / \partial t) s_{\lambda}\left(t_{0}, \tau\right)<0$. Now differentiate (1.4) with respect to $t$ and evaluate at $t=t_{0}$ to obtain

$$
\frac{\partial}{\partial t} s_{\lambda}\left(t_{0}, \tau\right)+\lambda a\left(t_{0}, t_{0}\right) s_{\lambda}\left(t_{0}, \tau\right)+\lambda \int_{\tau}^{t_{0}} \frac{\partial}{\partial t} a\left(t_{0}, u\right) s_{\lambda}(u, \tau) d \tau=0
$$

By (ii) we obtain $(\partial / \partial t) s_{\lambda}\left(t_{0}, \tau\right)>0$ unless $(\partial / \partial t) a\left(t_{0}, u\right)=0$ a.e. for $\tau<u<t_{0}$. But then we obtain

$$
(\partial / \partial t) s_{\lambda}(t, \tau)+\lambda a(t, t) s_{\lambda}(t, \tau)=0 \text { for } \tau<t<t_{0} .
$$

Solving for $s_{\lambda}(t, \tau)$ in (3.4) we obtain $s_{\lambda}\left(t_{0}, \tau\right)=C \exp \left(-\int_{\tau}^{t_{0}} a(u, u) d u\right)>0$, again a contradiction. This establishes (3.2). 
Next we wish to show that there are positive constants $C_{\alpha}$ for $0<\alpha<1$, independent of $(t, \tau)$, so that

$$
\sup _{\lambda>0} \lambda^{\alpha} r_{\lambda}(t, \tau)<C_{\alpha} a(t, \tau)(t-\tau)^{-\alpha}
$$

and

$$
\sup _{\lambda>0} \lambda^{\alpha} s_{\lambda}(t, \tau)<C_{\alpha}(t-\tau)^{-\alpha} .
$$

By Theorem 2.7 of [9] we can rewrite (1.3) as

$$
r_{\lambda}(t, u)=a(t, u)-\lambda \int_{u}^{t} r_{\lambda}(t, o) a(o, u) d o
$$

Integrating (3.7) in $u$ from $\tau$ to $t$ and interchanging the order of integration we obtain

$$
\int_{\tau}^{t} r_{\lambda}(t, u) d u=\int_{\tau}^{t} a(t, u) d u-\lambda \int_{\tau}^{t} r_{\lambda}(t, o)\left[\int_{\tau}^{o} a(o, u) d u\right] d o .
$$

By (iii) we have $\int_{\tau}^{o} a(o, u) d u$ is an increasing function of $o$ and since $r_{\lambda}(t, \tau)>0$ (3.8) implies

$$
\int_{\tau}^{t} r_{\lambda}(t, u) d u>\int_{\tau}^{t} a(t, u) d u-\lambda\left[\int_{\tau}^{t} a(t, u) d u\right]\left[\int_{\tau}^{t} r_{\lambda}(t, o) d o\right] .
$$

Solving (3.9) we get

$$
\int_{\tau}^{t} r_{\lambda}(t, u) d u>\left[\int_{\tau}^{t} a(t, u) d u\right]\left[1+\lambda \int_{\tau}^{t} a(t, u) d u\right]^{-1} .
$$

On the other hand the second part of (ii) implies

$$
\lambda \int_{\tau}^{t} r_{\lambda}(t, u) d u<\frac{\lambda \int_{\tau}^{t} r_{\lambda}(t, u) a(u, \tau) d u}{a(t, \tau)}=\frac{a(t, \tau)-r_{\lambda}(t, \tau)}{a(t, \tau)}
$$

and hence we have

$$
r_{\lambda}(t, \tau)<a(t, \tau)\left[1-\lambda \int_{\tau}^{t} r_{\lambda}(t, u) d u\right]
$$

Combining (3.10) and (3.11) we obtain

$$
r_{\lambda}(t, \tau)<a(t, \tau)\left[1+\lambda \int_{\tau}^{t} a(t, u) d u\right]^{-1} .
$$

(Note that (3.12) extends Theorem 1 of [3].) By (3.1) and (3.12) we obtain

$$
s_{\lambda}(t, \tau)<\left[1+\lambda \int_{\tau}^{t} a(t, u) d u\right]^{-1} \text {. }
$$

Now multiplying (3.12) and (3.13) by $\lambda^{\alpha}$, maximizing in $\lambda$ for $0<\alpha<1$ and using the first part of (ii) we obtain (3.5) and (3.6).

Define resolvent operators $R(t, \tau)$ and $S(t, \tau)$ by (1.5) and (1.6) respectively. By (3.5) and (3.6) we have

$$
\begin{aligned}
& \left\|A^{\alpha} R(t, \tau)\right\|<C_{\alpha} a(t, \tau)(t-\tau)^{-\alpha}, \quad 0<\alpha<1, \\
& \left\|A^{\alpha} S(t, \tau)\right\|<C_{\alpha}(t-\tau)^{-\alpha}, \quad 0<\alpha<1,
\end{aligned}
$$


where $\|\cdot\|$ is the operator norm. It follows easily from the continuity of $r_{\lambda}(t, \tau)$ and $s_{\lambda}(t, \tau)$ and the dominated convergence theorem that, for each $x \in H$, $A^{\alpha} R(t, \tau) x$ and $A^{\alpha} S(t, \tau) x$ are continuous in $(t, \tau)$. Next we shall show that

$$
\int_{\tau}^{t} a(t, u) A S(u, \tau) x d u=x-S(t, \tau) x
$$

and

$$
\int_{\tau}^{t} a(t, u) A R(u, \tau) x d u=a(t, \tau) x-R(t, \tau) x
$$

for $x \in D\left(A^{\alpha}\right)$ and $0<\alpha<1$.

To establish (3.16) note that $a(t, u) A S(u, \tau) x=a(t, u) A^{1-\alpha} S(u, \tau) A^{\alpha} x$ so that by (3.15) the integral in (3.16) makes sense. Hence if $y \in H$ we have

$$
\begin{aligned}
\left\langle\int_{\tau}^{t} a(t, u) A S(u, \tau) x\right. & d u, y\rangle=\int_{\tau}^{t} \int_{0}^{\infty} \lambda a(t, u) s_{\lambda}(u, \tau) d E_{x, y}(\lambda) d u \\
& =\int_{0}^{\infty} \int_{\tau}^{t} \lambda a(t, u) s_{\lambda}(u, \tau) d u d E_{x, y}(\lambda) \\
& =\int_{0}^{\infty}\left[1-s_{\lambda}(t, \tau)\right] d E_{x, y}(\lambda)=\langle x-S(t, \tau) x, y\rangle
\end{aligned}
$$

which establishes (3.16). The proof of (3.17) is similar.

To complete the proof of Theorem 1 we must show that if $x_{1}(t)=f_{1}(t)-$ $\int_{0}^{t} R(t, \tau) A f_{1}(\tau) d \tau$ and if $x_{2}(t)=S(t, 0) f_{2}(0)+\int_{0}^{t} S(t, \tau) f^{\prime}(\tau) d \tau$ then $x_{1}(t)$ and $x_{2}(t)$ satisfy (1.1). If $x_{1}(t)$ is as given above we have

$$
\begin{aligned}
\int_{0}^{t} a(t, \tau) A x_{1}(\tau) & d \tau=\int_{0}^{t} a(t, \tau) A f_{1}(\tau) d \tau-\int_{0}^{t} \int_{0}^{\tau} a(t, \tau) A R(\tau, u) A f_{1}(u) d u d \tau \\
& =\int_{0}^{t} a(t, \tau) A f_{1}(\tau) d \tau-\int_{0}^{t} \int_{u}^{t} a(t, \tau) A R(\tau, u) d \tau A f_{1}(u) d u \\
& =\int_{0}^{t} a(t, \tau) A f_{1}(\tau) d \tau-\int_{0}^{t} a(t, u) A f_{1}(u) d u+\int_{0}^{t} R(t, u) A f_{1}(u) d u \\
& =f_{1}(t)-x_{1}(t)
\end{aligned}
$$

by (3.17) and the fact that $A f_{1}(u) \in D\left(A^{\alpha}\right)$. Similarly we have

$$
\begin{aligned}
\int_{0}^{t} a(t, \tau) A x_{2}(\tau) d \tau= & \int_{0}^{t} a(t, \tau) A S(\tau, 0) f_{2}(0) d \tau \\
& +\int_{0}^{t} \int_{u}^{t} a(t, \tau) A S(\tau, u) d \tau f_{2}^{\prime}(u) d u \\
= & f_{2}(0)-S(t, 0) f_{2}(0)+\int_{0}^{t} f_{2}^{\prime}(u) d u-\int_{0}^{t} S(t, u) f_{2}^{\prime}(u) d u \\
= & f_{2}(t)-\left[S(t, 0) f_{2}(0)+\int_{0}^{t} S(t, u) f_{2}^{\prime}(u) d u\right] \\
= & f_{2}(t)-x_{2}(t)
\end{aligned}
$$

by (3.16) and the fact that $f_{2}^{\prime} \in D\left(A^{\alpha}\right)$. Now (2.1) follows immediately from this representation of solutions and uniqueness is proved exactly as in Lemma 2.3 of [1]. 
The proof of Theorem 2 only entails one major change in the proof of Theorem 1, namely establishing inequalities similar to (3.5) and (3.6) for $\alpha=0$. We now wish to show that

$$
\sup _{\lambda>0}\left|r_{\lambda}(t, \tau)\right|<2 a(\tau, \tau)
$$

and

$$
\sup _{\lambda>0}\left|s_{\lambda}(t, \tau)\right|<1 .
$$

We begin by replacing $t$ by $t+\tau$ in (1.3) and (1.4). A simple change of variable and the substitutions $\tilde{r}_{\lambda}(t)=r_{\lambda}(t+\tau, \tau), \tilde{s}_{\lambda}(t)=s_{\lambda}(t+\tau, \tau), \tilde{a}(t)=a(t+\tau, \tau)$ and $b(t, y)=a(t+\tau, y+\tau)$ allow us to rewrite (1.3) and (1.4) as

$$
\tilde{r}_{\lambda}(t)+\int_{0}^{t} \lambda b(t, y) \tilde{r}_{\lambda}(y) d y=\tilde{a}(t)
$$

and

$$
\tilde{s}_{\lambda}(t)+\int_{0}^{t} \lambda b(t, y) \tilde{s}_{\lambda}(y) d y=1 .
$$

The kernel $\lambda b(t, y)$ ( $\tau$ fixed) satisfies the hypotheses of Theorem 2 of [8] and hence we may conclude that

$$
\begin{aligned}
& \left|\tilde{r}_{\lambda}(t)\right|<2 a(\tau, \tau), \\
& \left|\tilde{s}_{\lambda}(t)\right| \leqslant 1 .
\end{aligned}
$$

This immediately establishes (3.21) and (3.22). Exactly as before one can show that (3.16) and (3.17) are valid for $x \in D(A)$ and hence the calculations in (3.19) and (3.20) are still valid under the more restrictive hypotheses on $f(t)$.

The uniqueness of the solution is proved as before with the following minor change. If we define $J_{n}=(I+A / n)^{-1}$ and $A_{n}=n\left(I-J_{n}\right)$ then $A_{n}$ is a bounded, positive, selfadjoint operator satisfying $A_{n}=A J_{n}$. If $\left\{E_{n}(\lambda) \mid \lambda>0\right\}$ is the resolution of the identity associated with $A_{n}$, then we have $d E_{n}(\lambda)=(1+\lambda / n)^{-1} d E(\lambda)$. Hence, if $R_{n}(t, \tau)$ is the resolvent operator given by $A_{n}$ (cf. (1.5)) then $R_{n}(t, \tau)=$ $J_{n} R(t, \tau)$. It follows immediately that $\int_{0}^{t} R_{n}(t, \tau) g(\tau) d \tau \rightarrow \int_{0}^{t} R(t, \tau) g(\tau) d \tau$ in $L^{1}[0, T ; H]$ for every $g \in L^{1}[0, T ; H]$. This fact, combined with the proof of Lemma 2.3 of [1], will establish uniqueness.

\section{REFERENCES}

1. Ph. Clément and J. A. Nohel, Abstract linear and nonlinear Volterra equations preserving pasitivity, SIAM J. Math. Anal. 10 (1979), 365-388.

2. A. Friedman and N. Shinbrot, Volterra integral equations in Banach space, Trans. Amer. Math. Soc. 126 (1967), 131-179.

3. G. Gripenberg, On positive, nonincreasing resolvents of Volterra equations, J. Differential Equations 30 (1978), 380-390.

4. On Volterra equations with nonconvolution kernels, Report HTKK-MAT-A118, Helsinki Univ. Technology, 1978.

5. , On a linear Volterra equations in a Hilbert space, Report HTKK-MAT-A127, Helsinki

Univ. Technology, 1978. 
6. K. Hannsgen, The resolvent kernel of an integro-differential equation in Hilbert space, SIAM J. Math. Anal. 7 (1976), 481-490.

7. T. Kiffe and M. Stecher, Properties and applications of the resolvent operator to a Volterra integral equation in Hilbert space, SIAM J. Math. Anal. 11 (1980), 82-91.

8. J. J. Levin, Remarks on a Volterra equation, delay and functional differential equations and their applications, Academic Press, New York, 1972, pp. 233-255.

9. R. K. Miller, Nonlinear Volterra integral equations, Benjamin, Menlo Park, California, 1971.

10. K. Yosida, Functional analysis, Springer-Verlag, Berlin and New York, 1974.

Department of Mathematics, Texas AdM University, College Station, Texas 77843 